\title{
Effect of Pioglitazone on Production of Regulated upon Activation Normal T-cell Expressed and Secreted (RANTES) and IVF Outcomes in Infertile Women with Endometriosis
}

\author{
${ }^{\dagger}$ Chung-Hoon Kim${ }^{1}$, You-Jeong Lee ${ }^{1}$ Jun-Bum Kim ${ }^{1}$, Kyung-Hee Lee ${ }^{1}$, Su-Kyung Kwon', \\ Jun-Woo Ahn', Sung-Hoon Kim ${ }^{1}$, Hee-Dong Chae ${ }^{1}$ and Byung-Moon Kang ${ }^{1}$ \\ ${ }^{1}$ Division of Reproductive Endocrinology and Infertility, Department of Obstetrics and Gynecology, \\ College of Medicine, University of Ulsan, Asan Medical Center, Seoul 138-736, Republic of Korea \\ ${ }^{2}$ Division of Reproductive Endocrinology and Infertility, Department of Obstetrics and Gynecology, College of \\ Medicine, University of Ulsan, Ulsan University Hospital, Ulsan 682-714, Republic of Korea
}

\begin{abstract}
This study was performed to investigate the effect of peroxisome proliferators activated receptor- $\gamma$ (PPAR$\gamma$ ) ligand, pioglitazone, on production of regulated upon activation normal T-cell expressed and secreted (RANTES) and in vitro fertilization (IVF) outcome in infertile patients with endometriosis. Sixty-four infertile patients with stage III or IV endometriosis undergoing IVF were randomly allocated to the study or the control group. The long protocol of GnRH agonist (GnRH-a) was used for controlled ovarian stimulation (COS) in all patients. Patients in the study group were treated with pioglitazone at a dose of $15 \mathrm{mg} /$ day orally from the starting day of GnRH-a treatment to the day of hCG injection. Blood samples were drawn for serologic assay of RANTES on the first day of GnRH-a treatment and the day of hCG injection. There were no differences between the study and control groups in patient characteristics. There were also no differences between the two groups in COS duration, and the numbers of retrieved oocytes, fertilized oocytes and embryos transferred. The clinical pregnancy rate per cycle was higher in the study group, but this difference was not statistically significant. However, embryo implantation rate was significantly higher in the study group of $12.5 \%$ compared with $8.6 \%$ in the control group $(P<0.05)$. The serum RANTES levels after pioglitazone treatment were significantly lower than those before pioglitazone treatmen in the study group $(P<0.05)$. Our data suggest that pioglitazone treatment can suppress RANTES production and improve the embryo implantation rate in patients with endometriosis undergoing IVF.
\end{abstract}

Key words : Controlled ovarian stimulation, Endometriosis, IVF, Pioglitazone, PPAR- $\gamma$ ligand, Regulated upon activation normal T-cell expressed and secreted

\section{INTRODUCTION}

Regulated upon activation normal T-cell expressed and secreted (RANTES) is a key signaling cytokine involved in initiation of physiologic inflammation (Conti et al., 1999) as well as in many pathologic inflammatory conditions including endometriosis (Oral et al., 1996). RANTES has been shown to be responsible for $70 \%$ of the stimulation of monocyte migration caused by peritoneal fluid from women with endometriosis (Hornung et al., 2001a). Some authors have suggested that RANTES production might be inhibited by activation of the PPAR pathway. Thia-

\footnotetext{
Manuscript received 19 August 2013, Received in revised form 25 August 2013, Accepted 30 August 2013

${ }^{\dagger}$ Corresponding Author : Chung-Hoon Kim, Division of Reproductive Endocrinology and Infertility, Department of Obstetrics and Gynecology, College of Medicine, University of Ulsan, Asan Medical Center, 88, Olympic-ro 43-gil, Songpa-gu, Seoul 138-736, Korea. Tel. : +82-2-3010-3639, Fax : +82-23010-6944, E-mail : chnkim@amc.seoul.kr

This is an Open Access article distributed under the terms of the Creative Commons Attribution Non-Commercial License(http://creativecommons.org/ licenses/by-nc/3.0) which permits unrestricted non-commercial use, distribution, and reproduction in any medium, provided the original work is properly cited.
} 
zolidinediones including rosiglitazone and pioglitazone are a new class of insulin sensitizers that do not stimulate insulin secretion (Adams et al., 1997). These drugs act as PPAR- $\gamma$ agonists, alter gene expression, enhance the sensitivity of tissue to insulin, and partially reverse lipid abnormalities in patients with diabetes (Lenhard, 2001). Therefore, theses drugs have been used for patients with polycystic ovary syndrome (PCOS) undergoing IVF treatment. Our previous study demonstrated that pioglitazone therapy reduces intraovarian stromal blood flow and is beneficial in improving both the response to ovarian stimulation and IVF outcome in PCOS patients undergoing IVF (Kim et al., 2010). It has been reported that PPAR- $\gamma$ also has a beneficial effect on endometriosis. In an in vitro study, PPAR- $\gamma$ reduced the monocyte migration induced by peritoneal fluid from women with endometriosis (Hornung et al., 2001b). Therefore, we performed this study to investigate the effect of pioglitazone, a drug of the thiazolidinedione class, on production of RANTES and in vitro fertilization-embryo transfer (IVF-ET) outcomes in infertile patients with stage II or IV endometriosis undergoing IVF treatment.

\section{MATERIAL AND METHODS}

\section{Patients}

Sixty-four infertile patients with stage III or IV endometriosis who underwent IVF were prospectively recruited to this randomized study, which was performed at the university-based infertility clinic of the Asan Medical Center, Seoul, South Korea. All subjects were diagnosed by laparoscopic surgery and histologic examination with stage III or IV endometriosis according to the revised American Society for Reproductive Medicine (ASRM) classification. All patients provided written informed consent. Patients were randomly allocated to the treatment group $(n=32)$ or the placebo group $(n=32)$ by number allocation using sealed envelopes. A power calculation showed $82 \%$ when each groups consisted of 32 patients. All patients had endometriosis, and had failed to ovulate or conceive after repeated treatment with clomiphene citrate or gonadotropin. All patients were in good health with normal thyroid, hepatic, and renal function. Blood samples were collected from each patient on menstrual cycle day (MCD) 3 for basal measurement of sex hormone and prolactin levels.

\section{Controlled Ovarian Stimulation (COS)}

The long protocol of GnRH agonist (GnRH-a) was used for COS in all patients. Daily injection of a GnRH agonist $1 \mathrm{mg}$ (Leuprorelin acetate, Lorelin; Dongkook, Korea) was initiated commencing at the midluteal phase and was continued until menses, followed by a dose reduction to $0.5 \mathrm{mg}$ daily. Ovarian stimulation was started with 50-150 IU of recombinant human follicle-stimulating hormone (rhFSH, Puregon; Organon, Oss, the Netherlands) on menstrual day 3. The initial dose of rhFSH was calculated with reference to patient age and body mass index. When one or more follicles attained a mean diameter of $\geq 18 \mathrm{~mm}, 250$ hCG was subcutaneously administered to induce follicular maturation.

In the pioglitazone treatment group, pioglitazone (Actos; Takeda, Osaka, Japan), 15 mg once daily, was given on the commencement day of GnRH-a administration to the day of human chorionic gonadotropin (hCG, Ovidrel; Serono, Geneva, Switzerland) injection. In the control group, placebo was administered during the same period. Oocytes were retrieved 35-36 hours later, and one to four embryos were transferred to the uterus three days later. During the luteal phase, $90 \mathrm{mg}$ of vaginal progesterone gel (Crinone 8\%; Serono) was applied once daily, starting on the day of oocyte retrieval. Pregnancies were identified by an increase in serum $\beta$-hCG concentration and transvaginal ultrasonographic evidence of a gestational sac. Serum concentrations of $\beta$-hCG were measured 11 days after embryo transfer (ET) by radioimmunoassay using an hCG MAIA clone kit (Serono Diagnostics, Woking, UK); the inter- and intra-assay variances values were $<10 \%$ and $5 \%$, respectively. A clinical pregnancy was defined as the presence of a gestational sac on ultrasonography. Blood 
samples were drawn for serologic assay of RANTES on the day on which the GnRH agonist was first administered and the day of HCG injection. We analyzed serum RANTES concentrations before and after treatment in both groups. RANTES was measured using a solid-phase enzyme-linked immunosorbent assay (Medgenix Diagnostics, Fleurus, Belgium); interassay and intraassay variances were less than $10 \%$ and $5 \%$, respectively..

\section{Statistical analysis}

Results are reported as means \pm standard deviations (SDs). The Chi-squared test, Fisher's exact test, the Mann-Whitney $U$ test and Spearman's coefficient of correlation were used, as appropriate, in comparisons. Statistical significance was defined as $P<0.05$. The SPSS statistical package for Windows, version 11.0 (SPSS Inc, Chicago, IL) was used for all analyses.

\section{RESULTS}

Patients' clinical characteristics and baseline hormone concentrations were similar in the pioglitazone and control groups (Table 1). Table 2 shows the COS results and
IVF-ET outcomes. There were no differences between the two groups in the total dose of rhFSH administered, and the numbers of oocytes retrieved, oocytes fertilized and grade I or II embryos. The clinical pregnancy rate was somewhat higher in the pioglitazone group of $37.5 \%$ compared with $31.3 \%$ of the control group, but the difference did not achieve the statistical significance. However, the embryo implantation rate was significantly higher in the pioglitazone group than in the control group (13.8\% vs. $8.6 \%, P<0.01)$. There was no significant difference between the two groups in the miscarriage rates.

When COS results and IVF outcomes were compared between pregnant and non-pregnant subgroups of the pioglitazone group, there were no significant differences between pregnant and non-pregnant subgroups in the numbers of oocytes retrieved, mature oocytes, oocytes fertilized and embryos transferred.

The serum RANTES concentrations of the pioglitazone and control groups are shown in Fig. 1. RANTES concentrations after pioglitazone treatment decreased significantly compared with that before pioglitazone treatment $(P<0.01)$. However, RANTES concentration before and after placebo treatment was not different in the control group. In the

Table 1. Patient characteristics

\begin{tabular}{|c|c|c|c|}
\hline & Treatment group & Control group & $P$ \\
\hline No. of patients & 32 & 32 & \\
\hline Age of patient (years) & $33.8 \pm 3.7$ & $33.5 \pm 4.1$ & NS \\
\hline Age of husband (years) & $39.2 \pm 7.2$ & $39.0 \pm 6.9$ & NS \\
\hline Infertility duration (years) & $3.7 \pm 2.2$ & $3.5 \pm 2.1$ & NS \\
\hline Patients with primary infertility (\%) & $37.5(12 / 32)$ & $31.3(10 / 32)$ & NS \\
\hline Body mass index $\left(\mathrm{kg} / \mathrm{m}^{2}\right)$ & $21.7 \pm 1.6$ & $21.5 \pm 1.4$ & NS \\
\hline Patients with endometriosis IV (\%) & $56.3(18 / 32)$ & $53.1(17 / 32)$ & NS \\
\hline Patients with endometrioma $>4 \mathrm{~cm}$ (\%) & $56.3(18 / 32)$ & $50.0(16 / 32)$ & NS \\
\hline Patients with bilateral endometriomas (\%) & $50.0(16 / 32)$ & $46.9(15 / 32)$ & NS \\
\hline \multicolumn{4}{|l|}{ Endocrine profile } \\
\hline 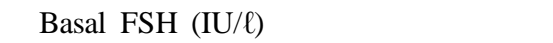 & $6.7 \pm 1.9$ & $6.5 \pm 1.7$ & NS \\
\hline 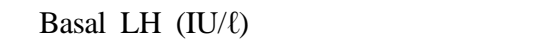 & $5.7 \pm 1.6$ & $5.8 \pm 2.1$ & NS \\
\hline Basal testosterone (ng/ ) & $0.5 \pm 0.2$ & $0.4 \pm 0.1$ & NS \\
\hline Prolactin (ng/ ) & $15.2 \pm 4.3$ & $14.6 \pm 3.9$ & NS \\
\hline
\end{tabular}

Note: Values are means \pm standard deviations. FSH, follicle-stimulating hormone; LH, luteinizing hormone; NS = not significant. 
Table 2. Comparison of controlled ovarian stimulation results and IVF-ET outcome

\begin{tabular}{|c|c|c|c|}
\hline & Treatment group & Control group & $P$ \\
\hline No. of cycles initiated & 32 & 32 & \\
\hline Total dose of rhFSH (IU) & $2,513.4 \pm 421.6$ & $2,491.3 \pm 398.3$ & NS \\
\hline Endometrial thickness on day of HCG administration (mm) & $10.2 \pm 1.6$ & $10.0 \pm 1.4$ & NS \\
\hline No. of oocytes retrieved & $7.9 \pm 3.1$ & $7.6 \pm 2.9$ & NS \\
\hline No. of MII oocytes retrieved & $6.6 \pm 2.7$ & $6.4 \pm 2.5$ & NS \\
\hline No. of oocytes fertilized & $6.3 \pm 2.6$ & $6.2 \pm 2.3$ & NS \\
\hline No. of grade I or II embryos produced & $3.8 \pm 2.3$ & $3.6 \pm 2.4$ & NS \\
\hline No. of embryos transferred & $3.6 \pm 0.5$ & $3.6 \pm 0.4$ & NS \\
\hline Clinical PR / cycle initiated (\%) & $37.5(12 / 32)$ & $31.3(10 / 32)$ & NS \\
\hline Multiple PR / clinical pregnancy (\%) & $33.3(4 / 12)$ & $20.0(2 / 10)$ & NS \\
\hline Embryo implantation rate (\%) & $13.8(16 / 116)$ & $8.6(10 / 116)$ & $<0.01$ \\
\hline Miscarriage rate (\%) & $8.3(1 / 12)$ & $16.7(2 / 10)$ & NS \\
\hline
\end{tabular}

Note: Values are means \pm standard deviations. rhFSH, recombinant human follicle-stimulating hormne; NS, not significant; PR, pregnancy rate

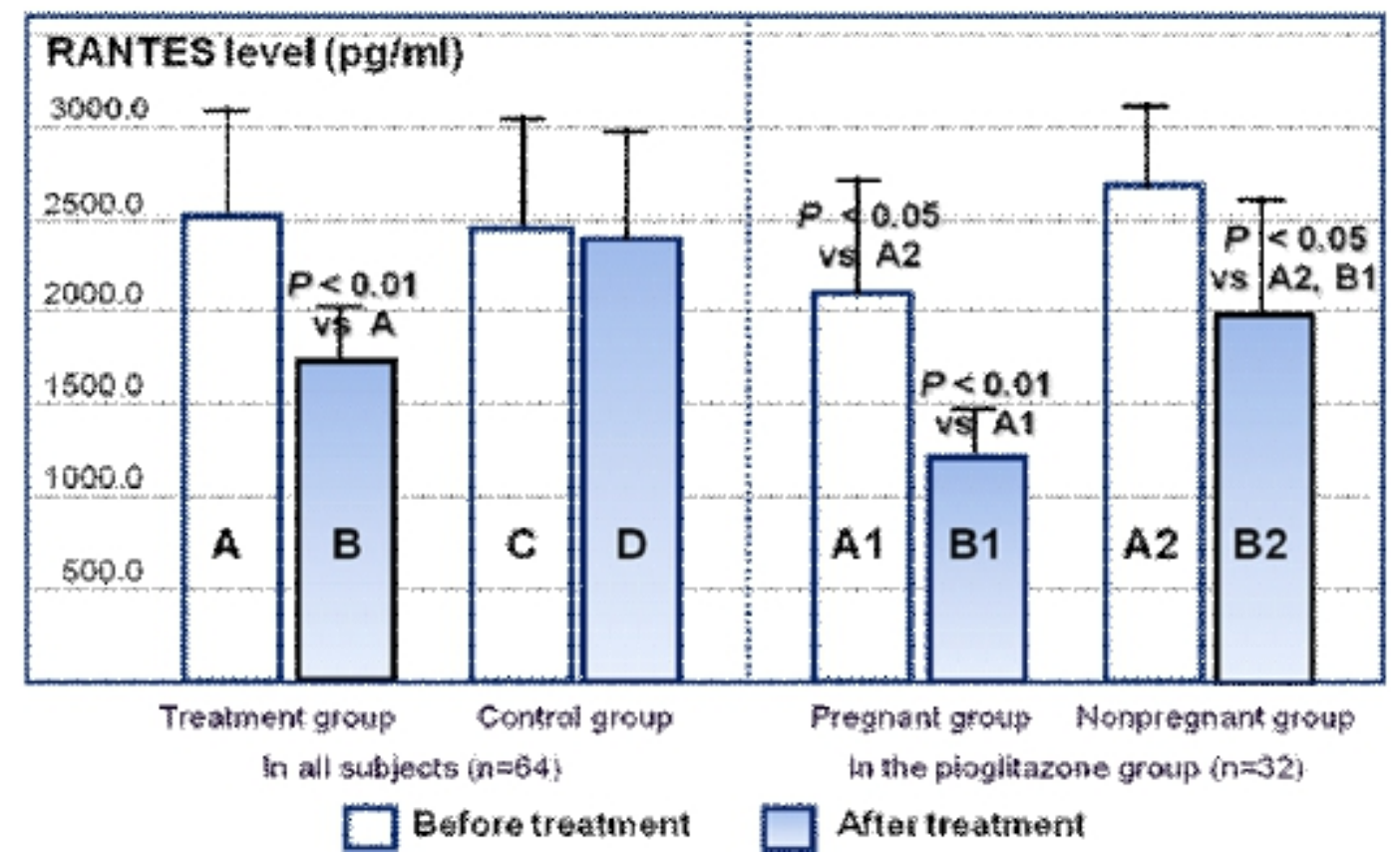

Fig. 1. Plasma RANTES levels in patients with endometriosis who underwent IVF-ET before and after pioglitazone treatment.

pregnant subgroup of the pioglitazone group, RANTES concentration before pioglitazone treatment was significantly lower than in the non-pregnant subgroup $(P<0.05)$. After pioglitazone treatment, RANTES concentrations decreased significantly in both subgroups $(P<0.01$ in the pregnant subgroup, $P<0.05$ in the non-pregnant subgroup).

\section{DISCUSSION}

Endometriosis is an inflammatory disease characterized by the presence of endometrial glandular and stromal cells in areas outside of the uterine corpus. The reported disease prevalence varies widely depending on the population 
studied and the method of sampling employed, the prevalence of endometriosis appears to range from $2 \%$ to $18 \%$, whereas within infertile populations the prevalence has been reported to be as high as 50\% (Missmer \& Cramer, 2003). Endometriosis is associated with activation of immune cells and aberrant cytokine production in peritoneal fluid, creating a local inflammatory environment (Lebovic et al., 2001; Wu \& Ho, 2003).

In pathologic states including chronic endometritis and endometriosis, inflammation followed by cellular infiltration into the area of interest and is followed by differentiation and activation of immune cells (Pritts et al., 2002). Such cells secrete growth factors, cytokines, prostanoids, complement components, and hydrolytic enzymes (Oral et al., 1996). These signals are released into the bloodstream and chemoattract other immune cells. The levels of cytokines associated with inflammation have been reported to be higher in women with endometriosis (Bedaiwy et al., 2002) as well as in those with unexplained infertility (Demir et al., 2009). Both ectopic and eutopic endometrium can be stimulated to produce materials chemoattracting mononuclear cells, including monocyte chemotactic protein-1 (MCP-1) (Arici et al., 1997), interleukin-8 (IL-8) (Arici et al., 1996) and RANTES (Khorram et al., 1993). Many studies have demonstrated abnormal local endometrial production of pro-inflammatory cytokines, such as tumor necrosis factor a (TNF-a), IL-1, IL-6, and IL-8 (Koninckx et al., 1999). Increasing evidence indicates that RANTES is the key chemokine involved at early stages of the inflammatory process (Appay \& Rowland-Jones, 2001). One report showed that RANTES is responsible for $70 \%$ of the in vitro monocyte migration activity in peritoneal fluid from women with endometriosis (Hornung et al., 2001). Subsequent activation of cellular inflammatory mediators might be prevented by blocking of early monocyte infiltration.

PPAR plays a key role in the regulation of inflammation and insulin secretion (Yessoufou et al., 2006). Komar et al. (2001) demonstrated transcription of PPAR- $\gamma$ in rat granulosa cells. As well as exerting multiple effects on insulin production, recently thiazolinediones have been shown to exhibit both insulin-independent and insulinsensitizing direct effects on steroidogenesis and production of IGF binding protein-1 in human ovarian cells. This implies that PPAR- $\gamma$ is expressed in the human ovary (Seto-Young et al., 2005). It has been reported that PPAR- $\gamma$ also affect RANTES secretion even in the endometrial stromal cells (Pritts et al., 2002). The cited in vitro study suggested that PPAR inhibits the pathway leading to RANTES expression in endometrial stromal cells. By binding to rosiglitazone, PPAR- $\gamma$ receptor-ligand complexes seem to interact with response elements on the RANTES gene promoter, thereby blocking the subsequent transcription and translation of RANTES (Pritts et al., 2002). However, studies on the effect of PPAR$\gamma$ or PPAR- $\gamma$ agonist on RANTES secretion in endometriosis or endometrial tissue are very limited. Moreover, it is hard to find out the study on the effect of PPAR- $\gamma$ agonist on RANTES and IVF outcome in patients with endometriosis.

Our study is the first report on the effect of thiazolinediones on RANTES secretion and IVF outcome in infertile women with advanced endometriosis undergoing IVF treatment. In the present study, we used a pioglitazone as a PPAR- $\gamma$ ligand to treat infertile patients with endometriosis and evaluated whether PPAR- $\gamma$ ligand such as pioglitazone could inhibit production of RANTES via activation of the PPAR pathway and improve IVF outcomes in infertile patients with advanced endometriosis undergoing IVF. As we expected before study, pioglitazone had a beneficial effect on RANTES secretion and IVF outcome in advanced enometriosis patients.

The thiazolidinediones including rosiglitazone and pioglitazone belong to a new class of insulin sensitizers that do not stimulate insulin secretion. The first available drug of the thiazolidinedione class, troglitazone, was effective to induce ovulation in patients with polycystic ovary syndrome (PCOS), but was later reported to be associated with fatal hepatotoxicity (Azziz et al., 2001). This caused the U.S. Food and Drug Administration 
(FDA) to withdraw approval of the drug. Rosiglitazone, another thiazolidinedione, has also been shown to be effective in inducing ovulation in PCOS patients. Few side-effects are evident in healthy subjects. However, the FDA encourages healthcare professionals to follow current recommendations on the drug label, including a contraindication of rosiglitazone use in patients with NYHA Class III or IV heart failure, and avoidance of use in patients with symptomatic heart failure. All patients should be monitored for signs and symptoms of heart failure (rapid weight gain, difficulty in breathing, and/or swelling) after initiation of treatment and after an increase in dose (Lago et al., 2007; Singh et al., 2007).

We have shown that administration of pioglitazone reduced the serum concentration of RANTES. Although the response to ovarian stimulation did not improve, the implantation rate was significantly higher in the treatment group. Further, in the pregnant subgroup, RANTES concentration was significantly lower than in the non-pregnant subgroup. Statistically significant differences in RANTES concentrations were evident before and after treatment. However, However, our study has a limitation to evaluate the efficacy of pioglitazone treatment in endometriosis patients due to a small number of sample available. Therefore, well-designed larger studies with longer followup times are needed to confirm the effectiveness and safety of pioglitazone.

\section{REFERENCES}

Adams M, Montague CT, Prins JB, Holder JC, Smith SA, Sanders L, Digby JE, Sewter CP, Lazar MA, Chatterjee VK, O'Rahilly S (1997) Activators of peroxisome proliferator-activated receptor gamma have depot-specific effects on human preadipocyte differentiation. J Clin Invest 100:3149-3153.

Appay V, Rowland-Jones SL (2001) RANTES: a versatile and controversial chemokine. Trends Immunol 22:8387.

Arici A, Tazuke SI, Attar E, Kliman HJ, Olive DL (1996)
Interleukin-8 concentration in peritoneal fluid of patients with endometriosis and modulation of interleukin-8 expression in human mesothelial cells. Mol Hum Reprod 2:40-45.

Arici A, Oral E, Attar E, Tazuke SI, Olive DL (1997) Monocyte chemotactic protein-1 concentration in peritoneal fluid of women with endometriosis and its modulation of expression in mesothelial cells. Fertil Steril 67: 1065-1072.

Azziz R, Ehrmann D, Legro RS, Whitcomb RW, Hanley R, Fereshetian AG, O'Keefe M, Ghazzi MN (2001) Troglitazone improves ovulation and hirsutism in the polycystic ovary syndrome: a multicenter, double blind, placebo-controlled trial. J Clin Endocrinol Metab 86: 1626-1632.

Bedaiwy MA, Falcone T, Sharma RK, Goldberg JM, Attaran M, Nelson DR, Agarwal A (2002) Prediction of endometriosis with serum and peritoneal fluid markers: a prospective controlled trial. Hum Reprod 17:426-431.

Conti P, Barbacane RC, Reale M (1999) Chemokines in inflammatory states. Allergy Asthma Proc 20:205-208.

Demir B, Guven S, Guven ES, Atamer Y, Gul T (2009) Serum IL-6 level may have role in the pathophysiology of unexplained infertility. Am J Reprod Immunol 62:261-267.

Hornung D, Bentzien F, Wallwiener D, Kiesel L, Taylor RN (2001a) Chemokine bioactivity of RANTES in endometriotic and normal endometrial stromal cells and peritoneal fluid. Mol Hum Reprod 7:163-168.

Hornung D, Waite LL, Ricke EA, Bentzien F, Wallwiener D, Taylor RN (2001b) Nuclear peroxisome proliferatoractivated receptors alpha and gamma have opposing effects on monocyte chemotaxis in endometriosis. J Clin Endocrinol Metab 86:3108-3114.

Khorram O, Taylor RN, Ryan IP, Schall TJ, Landers DV (1993) Peritoneal fluid concentrations of the cytokine RANTES correlate with the severity of endometriosis. Am J Obstet Gynecol 169:1545-1549.

Kim CH, Jeon GH, Kim SR, Kim SH, Chae HD, Kang BM (2010) Effects of pioglitazone on ovarian stromal 
blood flow, ovarian stimulation, and in vitro fertilization outcome in patients with polycystic ovary syndrome. Fertil Steril 94:236-241.

Koninckx PR, Kennedy SH, Barlow DH (1999) Endometriotic disease: the role of peritoneal fluid. Hum Reprod Update 4:741-751.

Komar CM, Braissant O, Wahli W, Curry TE Jr (2001) Expression and localization of PPARs in the rat ovary during follicular development and the periovulatory period. Endocrinol 142:4831-4838.

Lago RM, Singh PP, Nesto RW (2007) Congestive heart failure and cardiovascular death in patients with prediabetes and type 2 diabetes given thiazolidinediones: a meta-analysis of randomised clinical trials. Lancet 370:1129-1136.

Lebovic DI, Mueller MD, Taylor RN (2001) Immunobiology of endometriosis. Fertil Steril 75:1-10.

Lenhard JM (2001) PPAR gamma/RXR as a molecular target for diabetes. Receptors Channels 7:249-258.

Missmer SA, Cramer DW (2003) The epidemiology of endometriosis. Obstet Gynecol Clin North Am 30:119.

Oral E, Olive DL, Arici A (1996) The peritoneal environ- ment in endometriosis. Hum Reprod Update 2:385-398. Pritts EA, Zhao D, Ricke E, Waite L, Taylor RN (2002) PPAR-gamma decreases endometrial stromal cell transcription and translation of RANTES in vitro. J Clin Endocrinol Metab 87:1841-1844.

Seto-Young D, Paliou M, Schlosser J, Avtanski D, Park A, Patel P, Holcomb K, Chang P, Poretsky L (2005) Direct thiazolidinedione action in the human ovary: insulin-independent and insulin-sensitizing effects on steroidogenesis and insulin-like growth factor binding protein-1 production. J Clin Endocrinol Metab 90: 6099-6105.

Singh S, Loke YK, Furberg CD (2007) Long-term risk of cardiovascular events with rosiglitazone: a metaanalysis. JAMA 298:1189-1195.

Wu MY, Ho HN (2003) The role of cytokines in endometriosis. Am J Reprod Immunol 49:285-296.

Yessoufou A, Hichami A, Besnard P, Moutairou K, Khan NA (2006) Peroxisome proliferator-activated receptor alpha deficiency increases the risk of maternal abortion and neonatal mortality in murine pregnancy with or without diabetes mellitus: Modulation of $\mathrm{T}$ cell differentiation. Endocrinology 147:4410-4418. 